\title{
Dietary garlic and onion reduce the incidence of atherogenic diet-induced cholesterol gallstones in experimental mice
}

\author{
Satyakumar Vidyashankar, Kari Sambaiah and Krishnapura Srinivasan* \\ Department of Biochemistry and Nutrition, Central Food Technological Research Institute (CSIR), Mysore 570 020, India
}

(Received 22 April 2008 - Revised 18 August 2008 - Accepted 15 September 2008 - First published online 5 November 2008)

Mice fed with diet containing $0.5 \%$ cholesterol for 10 weeks resulted in cholesterol supersaturation in gallbladder bile which promoted the formation of cholesterol gallstones (CGS). In this study, dietary hypocholesterolaemic spices, garlic and onion (both raw or heat-processed) were examined for their antilithogenic potential by including at 0.6 and $2.0 \%$ level, respectively, along with lithogenic (LG) diet for 10 weeks. Dietary garlic and onion reduced the CGS incidence by $15-39 \%$, the effect being maximum in the heat-processed onion group. Dietary garlic and onion markedly reduced biliary cholesterol. The cholesterol:phospholipid ratio which was 1.58 in the LG diet group was reduced to $0.73-0.96$ in the garlic and onion groups. The biliary cholesterol saturation index was 0.92, 1.25, 1.09 and 0.86, respectively, in the heat-processed onion, raw garlic, heat-processed garlic and raw onion groups, while it was 1.9 in the LG group. The hydrophobicity index of bile was $-0.08,-0.079$, -0.032 and -0.073 , respectively, in the heat-processed onion, raw garlic, heat-processed garlic and raw onion groups, while it was +0.054 in the LG group. Hepatic hydroxymethyl glutaryl-CoA reductase activity was lowered in the LG diet-fed group, while dietary garlic or onion countered this alteration and also increased the activities of hepatic cholesterol $7 \alpha$-hydroxylase and sterol 27-hydroxylase. Serum and liver cholesterol were decreased by feeding garlic or onion compared to the LG diet. Thus, dietary Allium spices exerted antilithogenic influence by decreasing the cholesterol hyper-secretion into bile and increasing the bile acid output thus decreasing the formation of lithogenic bile in experimental mice.

Cholesterol gallstones: Cholesterol saturation index: Hydrophobicity index: Dietary garlic: Dietary onion

Cholesterol gallstone (CGS) disease is a highly prevalent gastroenteronological disorder resulting from alteration in hepatic and biliary cholesterol homeostasis. CGS disease is a multifactorial disease involving both environmental as well as genetic factors ${ }^{(1,2)}$. Gallstones formed in the gallbladder consist of cholesterol, bilirubin and calcium salts. The majority of gallstones are contributed by cholesterol and very small numbers of gallstones are primarily composed of calcium salts of bilirubin and phosphate. The pathogenic conditions that generally precede the occurrence of CGS are lithogenic bile, gallbladder stasis and short nucleation time. Lithogenicity of bile is determined by relative concentrations of three main components namely bile acids, phospholipid and cholesterol. Generally, lithogenic bile occurs with disruption of cholesterol homeostasis, leading to increased cholesterol secretion and subsequent supersaturation of bile with cholesterol ${ }^{(3,4)}$. But the molecular origins of altered secretion rates of biliary lipids in human cholesterol cholelithiasis remain unknown. Whether defects exist in hepatic lipid regulatory enzymes, responsible for cholesterol and bile acid biosynthesis, is not clear. Biliary bile acids are excreted through faeces and a major portion of these bile acids are reabsorbed in the intestine and assimilated by enterohepatic circulation. The decreased cholesterol excretion from the body and its increased biosynthesis and secretion into bile may increase the risk of CGS incidence.

Several dietary components are known to influence CGS. Diets containing animal protein such as casein favour CGS formation rather than diets containing soya protein ${ }^{(5)}$. Similarly, animals on diet containing polyunsaturated fat such as fish oil have a lower incidence of CGS compared to those on diets with saturated fat ${ }^{(6)}$. It is very well known that dietary fibre is beneficial in preventing CGS formation ${ }^{(7,8)}$. Very little information is available on the role of spices in CGS formation.

Spices are valued for their organoleptic as well as medicinal properties. Spices or their active principles like capsaicin from red pepper, curcumin from turmeric, fenugreek, garlic and onion have been widely documented for their hypocholesterolaemic as well as lipid-lowering properties ${ }^{(9)}$. Previously, it has been demonstrated that dietary curcumin (0.5\%) and capsaicin $(0.015 \%)$ were effective in inhibiting CGS formation and also in regression of pre-formed CGS in experimental mice ${ }^{(10,11)}$. Since formation of CGS in the gallbladder is preceded by supersaturation of bile with cholesterol, these hypocholesterolaemic spices may serve as potential dietary adjuncts to reduce the incidence of CGS and hence it is

Abbreviations: CGS, cholesterol gallstones; C/PL ratio, cholesterol:phospholipid ratio; CSI, cholesterol saturation index; HMG, hydroxymethyl glutaryl; LG diet, lithogenic diet.

* Corresponding author: Dr Krishnapura Srinivasan, fax +91821 2517233, email ksri.cftri@gmail.com 
very relevant to study their effect during lithogenic (LG) dietinduced CGS. In the present study, the two Allium spices, garlic and onion, were studied for their antilithogenic activity in experimental mice. Spices are prone to lose their bioactive principles during food processing conditions such as cook$\mathrm{ing}^{(12)}$. In this context, the antilithogenic potential of garlic and onion was evaluated both in their raw and heat-processed forms during LG diet-induced CGS formation in mice.

\section{Experimental methods}

\section{Materials}

Cholesterol, bile salts, dipalmitoyl phosphatidylcholine, boron trifluoride in methanol, bovine serum albumin, EDTA, hydroxymethyl glutaryl (HMG)-CoA, $3 \alpha$-hydroxy steroid dehydrogenase, NAD, standard bile acids kit (conjugated and unconjugated), Tris, triolein, and $\alpha$-cellulose were purchased from Sigma Chemical Co. (St Louis, MO, USA). Heparin and $\mathrm{MnCl}_{2}$ were obtained from SISCO Research Laboratory (Mumbai, India). Casein was purchased from Nimesh Corporation (Mumbai, India). All solvents used were of analytical grade and were distilled prior to use. Freeze-dried garlic and onion powders were a generous gift from Indo Nissin Food Ltd (Bangalore, India).

\section{Animal diets}

The animals were fed with AIN-76 semi-purified diet. The basal control diet consisted of: sucrose, $65 \%$; casein, $20 \%$; cellulose, 5\%; AIN-76 mineral mix, 3.5\%; AIN-76 vitamin mix, $1 \%$; DL-methionine, $0.3 \%$; choline chloride, $0.2 \%$ and refined peanut oil, $5 \%$. LG diet was prepared by supplementing $0.5 \%$ cholesterol and $0.25 \%$ bile salts (1:1 mixture of sodium cholate and sodium deoxycholate) to the AIN-76 basal diet. The diets were made isoenergetic by varying sucrose concentration. Various test diets were prepared by adding garlic powder $(0.6 \%)$ or onion powder $(2 \%)$ to the AIN-76 basal diet. The diets were made isocaloric by varying the sucrose content. Heat processing of garlic and onion was done by adding the respective freeze-dried powder to the boiling water and boiled for $15 \mathrm{~min}$ with constant stirring as practised normally in Indian cooking and used as heat-processed spices in these experiments. All these diets were prepared by mixing the ingredients in a mechanical mixer and pellets were prepared using a hand-operated pelletizer. Diets were stored at $4^{\circ} \mathrm{C}$ in air-tight containers.

\section{Animal treatment}

Animal experiments were carried out taking appropriate measures to minimize pain or discomfort in accordance with the guidelines laid down by the US National Institutes of Health regarding the care and use of animals for experimental procedures and with due clearance from the Institutional Animal Ethics Committee. Four-week-old male albino mice (108 mice; OUTB/Swiss Albino/IND/CFT (2c)) weighing 22 (SD 2) g were grouped and housed three mice per cage in polypropylene cages with sawdust as bedding. Groups of animals were fed ad libitum with LG diet and various test diets and had free access to water throughout the experimental period for 10 weeks. Body weights were recorded at weekly intervals. At the end, animals were fasted overnight and killed under ether anaesthesia. Blood was drawn immediately by cardiac puncture and the serum was separated by centrifugation for further analysis. The other organs were quickly excised, washed with ice-cold saline and blotted dry, weighed and stored at $-20^{\circ} \mathrm{C}$ till further analysis. Cholecystectomy was performed and gallbladders were carefully collected. The volume of bile was noted and the weight of gallbladder along with stones was recorded. The gallbladders were evaluated for CGS under magnifying lens for the presence of gallstones by four individuals unaware of dietary treatments. The grading of stones was done on a four-point scale ${ }^{(10)}$. The bile from the gallbladders was pooled and stored at $-20^{\circ} \mathrm{C}$ till further analysis.

\section{Analysis of lipids}

Gallstones were crushed to powder along with the fluid bile. Such a suspension of crushed stones in the bile was used for lipid extraction. Biliary lipids were extracted by the method of Bligh \& Dyer ${ }^{(13)}$ and the chloroform phase was used for lipid analysis. The upper methanolic phase was used for the estimation of total bile acids using $3 \alpha$-hydroxysteroid dehydrogenase $^{(14)}$. Serum and liver lipids were extracted by the method of Folch et al. ${ }^{(15)}$. Cholesterol levels were quantitated by the method of Searcy \& Bergquist ${ }^{(16)}$. Phospholipids were measured by the method of Charles \& Stewart ${ }^{(17)}$ using dipalmitoyl phosphatidylcholine as reference standard. TAG was estimated by the method of Fletcher ${ }^{(18)}$. Conjugated and unconjugated bile acids were quantified according to Rosi et al. ${ }^{(19)}$. The average hydrophobic and hydrophilic balance of biliary bile salts was quantified by means of a bile salt monomeric hydrophobicity index ${ }^{(20)}$. The cholesterol saturation index (CSI) of bile was calculated according to Carey ${ }^{(21)}$.

Fatty acids were analysed as methyl esters prepared using boron trifluoride in methanol as described by Morrison \& Smith $^{(22)}$ and analysed by GC (Shimadzu 14B, fitted with free induction decay) using a fused silica capillary column ( $25 \mathrm{~m} \times 0.25 \mathrm{~mm}$; Parma bond FFAP-DF-0.25; MacheryNagel GmbH Co., Duren, Germany). The operating conditions were: initial column temperature $160^{\circ} \mathrm{C}$, injector temperature $210^{\circ} \mathrm{C}$ and detector temperature $250^{\circ} \mathrm{C}$ : column temperature was programmed to rise at $6^{\circ} \mathrm{C}$ per min to the final temperature of $240^{\circ} \mathrm{C}$. Nitrogen gas was used as the carrier. Individual fatty acids were identified by comparing with retention times of standards (NU-Check Prep. Inc., Elysian, MN, USA) and were quantitated by an online choromatopack CR-6A integrator.

\section{Activities of enzymes of cholesterol metabolism}

HMG-CoA reductase activity in liver was assayed by following the formation of $\mathrm{CoA}^{(23)}$. Activities of cholesterol $7 \alpha$-hydroxylase and sterol 27-hydroxylase in liver were assayed as described by Petrack \& Latario ${ }^{(24)}$. Biliary proteins and protein in liver were analysed according to Lowry et al. ${ }^{(25)}$.

\section{Statistical analysis}

Statistical analysis was carried out using Graph Pad Prism statistical software (Graph Pad Prism, San Diego, CA, USA). 
Results are analysed by one-way ANOVA and the significance level was calculated using the Tukey-Kramer multiple comparison test and results are considered as significant at $P<0 \cdot 01$.

\section{Results}

Effect of feeding garlic and onion on the incidence of cholesterol gallstones during experimental induction

Effect of the dietary spices, garlic and onion, on CGS induction in mice was carried out by feeding LG diet with or without garlic or onion for a period of 10 weeks. Animals fed with control diet showed no sign of any cholesterol crystal or stone in the gallbladder, whereas all the animals in LG diet group showed CGS (Table 1). Animals fed with LG diet supplemented with garlic or onion showed significant reduction in the incidence of CGS. The percentage incidence of CGS was calculated by taking the incidence in LG diet as $100 \%$. The lowest incidence of CGS $(61.2 \%)$ was in the heat-processed onion group. Both spices, whether raw or heat-processed, were effective in reducing the formation of CGS, the reduction in CGS in experimental animals ranging from 14.5 to $38.8 \%$. The gallbladder size and weight were significantly increased by feeding lithogenc diet and dietary garlic and onion significantly countered increase in both gallbladder size and its weight (Table 1).

There were no significant differences in the body weight of animals between various diet groups at the end of the 10-week feeding period (Table 2). The liver weight was higher in the LG diet group. Kidney and heart weights were also increased significantly upon feeding LG diet and dietary spices countered this increase in kidney and heart weights.

\section{Effect of feeding garlic and onion on serum lipids during cholesterol gallstone induction}

The serum lipid profile in animals fed garlic or onion during experimental induction of CGS is presented in Table 3. The LG diet feeding caused hypercholesterolaemia where serum cholesterol increased 2-4-fold compared to control animals. Feeding of garlic or onion along with LG diet prevented the rise in serum cholesterol levels by 33, 29, 31 and $34 \%$ in

Table 1. Effect of feeding garlic and onion on cholesterol gallstone (CGS) incidence in mice

(Mean values and standard deviations for eighteen mice per group)

\begin{tabular}{|c|c|c|c|c|c|}
\hline \multirow[b]{2}{*}{ Diet group } & \multicolumn{2}{|c|}{$\begin{array}{l}\text { Gallbladder } \\
\text { weight }(\mathrm{mg})\end{array}$} & \multicolumn{2}{|c|}{ CGS score§ } & \multirow[b]{2}{*}{ CGS incidence (\% } \\
\hline & Mean & SD & Mean & SD & \\
\hline Control & $25 \cdot 5$ & 4.87 & 0.00 & & 0.0 \\
\hline LG & $67 \cdot 3^{*}$ & 4.38 & $3.40^{*}$ & 0.80 & $100 \cdot 0$ \\
\hline $\mathrm{LG}+$ garlic $(\mathrm{R})$ & 43.3† & 2.53 & $3.05 \dagger$ & 0.84 & $73 \cdot 2$ \\
\hline$L G+$ garlic $(H)$ & $38.5 \dagger$ & 3.55 & $3.02 \dagger$ & 0.71 & $85 \cdot 5$ \\
\hline $\mathrm{LG}+$ onion $(\mathrm{R})$ & $36.9 \dagger$ & 3.99 & $2.33 \dagger$ & $1 \cdot 15$ & $75 \cdot 2$ \\
\hline$L G+$ onion $(H)$ & $30.0 \dagger$ & 1.75 & $1.85 \dagger$ & 1.27 & $61 \cdot 2$ \\
\hline
\end{tabular}

$H$, heat-processed; LG, lithogenic; $R$, raw.

Mean values were significantly different from those of the control group: ${ }^{\star} P<0.01$

Mean values were significantly different from those of the LG group: $\dagger P<0.01$.

$\ddagger$ For details of diets and procedures, see Experimental methods.

$\S$ Scoring was done by four individuals and grading was done on a five-point scale (0-4). garlic (raw), garlic (heat-processed), onion (raw) and onion (heat-processed) groups, respectively. The phospholipid content of serum was significantly reduced by LG diet, whereas feeding of Allium spices significantly prevented this decrease in phospholipid content. The TAG content was significantly higher in the LG group compared to the control group. Feeding of heat-processed onion countered this increase in TAG. Feeding of LG diet significantly increased the cholesterol: phospholipid (C/PL) ratio and the Allium spices significantly reduced the increased $\mathrm{C} / \mathrm{PL}$ ratio.

\section{Effect of feeding garlic and onion on liver lipids during cholesterol gallstones induction}

Liver lipid profile in animals fed garlic or onion during experimental CGS induction is given in Table 4. The total cholesterol content was increased in LG diet group by $2 \cdot 8$ fold compared to control group. Feeding of Allium spices along with LG diet significantly countered this increase in hepatic cholesterol content by 25, 15, 15 and $30 \%$ in garlic (raw), garlic (heat-processed), onion (raw), and onion (heat-processed) groups respectively. Hepatic phospholipid content was decreased in LG diet group compared to control animals and this reduction was effectively countered in all spice fed groups. The increased $\mathrm{C} / \mathrm{PL}$ ratio as a result of LG diet was brought down significantly by spice feeding. Feeding of LG diet increased TAG content of liver and inclusion of spices in the diet significantly countered the raise in TAG content.

\section{Effect of feeding garlic and onion on biliary lipid profile during cholesterol gallstones induction}

The biliary lipid profile in animals fed Allium spices in addition to $\mathrm{LG}$ diet is given in Table 5. LG diet increased the cholesterol content of bile by six-fold. Dietary garlic or onion countered the increase in biliary cholesterol content significantly. The reduction ranged from 35 to $66 \%$ in various experimental groups compared to LG diet group. Phospholipids are one of the major constituents of bile, which help the bile to be physiologically active. Any disturbance or alteration in the phospholipid and bile acids results in the precipitation of cholesterol in bile. Phospholipid concentration in bile was increased in the LG group by $72 \%$ compared to control. Garlic (raw) and onion (heat-processed) feeding along with LG diet countered this increase in phospholipid content by 13 and $26 \%$, respectively. In the other two experimental groups (heat-processed garlic and raw onion) there was a marginal increase in phospholipid content. Bile salts form a major part of the bile solids. They are synthesized from the parent molecule cholesterol. The majority of the cholesterol is excreted from the system after converting into bile acids. Increase in the bile acid synthesis and excretion in bile tends to decrease the cholesterol content and lithogenicity of bile. The bile acid content in the bile remained essentially unaltered in the LG group and in groups supplemented with Allium spices.

In order to evaluate the relevance of the bile lipid profile in CGS formation, the C/PL ratio and cholesterol:bile acid ratio in bile were calculated (Table 5). The higher ratios of $\mathrm{C} / \mathrm{PL}$ and cholesterol:bile acid are the indicators of lithogenic bile 
Table 2. Effect of feeding garlic and onion on body and organ weights in miceł (Mean values and standard deviations for eighteen mice per group)

\begin{tabular}{|c|c|c|c|c|c|c|c|c|c|c|}
\hline \multirow[b]{2}{*}{ Diet group } & \multicolumn{2}{|c|}{ Body (g) } & \multicolumn{2}{|c|}{ Liver (g) } & \multicolumn{2}{|c|}{ Kidney (mg) } & \multicolumn{2}{|c|}{ Heart (mg) } & \multicolumn{2}{|c|}{ Spleen (mg) } \\
\hline & Mean & SD & Mean & SD & Mean & SD & Mean & SD & Mean & SD \\
\hline Control & 41.4 & $5 \cdot 82$ & 1.61 & 0.39 & 411.8 & 8.57 & $136 \cdot 0$ & $3 \cdot 82$ & $105 \cdot 7$ & $8 \cdot 27$ \\
\hline LG & $46 \cdot 9^{*}$ & 3.03 & $2 \cdot 90^{\star}$ & 0.19 & $518 \cdot 7^{\star}$ & $17 \cdot 6$ & $158 \cdot 5^{\star}$ & $6 \cdot 27$ & $106 \cdot 9$ & 4.94 \\
\hline LG + garlic $(R)$ & $41 \cdot 7$ & 1.56 & $2 \cdot 62$ & 0.19 & $427 \cdot 2 \dagger$ & 5.04 & $136 \cdot 5 \dagger$ & 3.67 & $100 \cdot 0$ & 3.55 \\
\hline$L G+$ garlic $(H)$ & 43.0 & 3.77 & $2 \cdot 74$ & 0.19 & $401.8 \dagger$ & $4 \cdot 31$ & $149 \cdot 3$ & 1.46 & $105 \cdot 0$ & 4.48 \\
\hline$L G+$ onion $(R)$ & 39.5 & 3.52 & 2.54 & 0.30 & $338.9 \dagger$ & 5.85 & $122 \cdot 1 \dagger$ & 3.99 & 91.7 & $4 \cdot 18$ \\
\hline$L G+$ onion $(H)$ & 43.3 & 1.93 & 2.55 & 0.14 & $384.8 \dagger$ & $2 \cdot 30$ & $142.9+$ & 1.98 & $96 \cdot 1$ & 1.56 \\
\hline
\end{tabular}

$\mathrm{H}$, heat-processed; LG, lithogenic; $\mathrm{R}$, raw.

Mean values were significantly different from those of the control group: ${ }^{\star} P<0.01$.

Mean values were significantly different from those of the $L G$ group: $\dagger P<0.01$.

$\ddagger$ For details of diets and procedures, see Experimental methods.

and it is very important to keep the cholesterol in soluble form. The effect of feeding garlic and onion on biliary lipids and biliary CSI during CGS induction in mice is presented in Table 6 . The C/PL ratio was 1.58 in LG diet-fed animals compared to 0.45 in the control group. The $\mathrm{C} / \mathrm{PL}$ ratio was brought down to $0.74,0.96,0.84$ and 0.73 , respectively, in raw garlic, heat-processed garlic, raw onion and heatprocessed onion diet groups. The cholesterol:bile acid ratio was 0.15 in $\mathrm{LG}$ diet-fed animals compared to 0.03 in the control group. The cholesterol:bile acid ratio was brought down to $0.06,0.10,0.08$ and 0.05 , respectively, in raw garlic, heat-processed garlic, raw onion and heat-processed onion diet groups.

The mole percentage of various bile acids present in the gallbladder bile of mice fed LG diet and test diets are given in Table 6. In the LG diet group, the taurocholic acid (TC) content was increased compared to the control group. The hydrophobic bile acids such as taurochenodeoxycholic acid and taurodeoxycholic acid content were increased and the hydrophilic bile acids such as tauromuricholic acid (TMC) and tauroursodeoxycholic acid were decreased in the LG diet-fed group. On the other hand, feeding Allium spices countered the decrease in the content of the hydrophilic bile acids tauromuricholic acid and tauroursodeoxycholic acid and the increase in hydrophobic bile acids taurochenodeoxycholic acid and taurodeoxycholic acid brought about by LG diet.

The hydrophobicity index is the net sum of hydrophobic and hydrophilic bile acids present in the bile. The hydrophobicity index was $-0 \cdot 145$ in the bile of control rats and it was increased to +0.054 in the bile of the LG diet group. Feeding of garlic (raw and heat-processed) or onion (raw or heatprocessed) along with LG diet decreased the hydrophobicity index of bile to $-0.079,-0.032,-0.073$ and -0.088 , respectively (Table 7). The CSI of the bile is a measure of supersaturation of bile with cholesterol. It was significantly higher in the LG group (1.90 compared to 0.51 in control) and feeding of Allium spices brought down CSI by 55, 43, 34 and $52 \%$, respectively, in raw garlic, heat-processed garlic, raw onion and heat-processed onion diet groups (Table 7).

Biliary fatty acid composition which reflects the type of fatty acids present in the biliary phospholipids is given in Table 8. Oleic acid was significantly decreased whereas palmitic and linoleic acids were increased in the LG group compared to controls. Dietary onion (either heat-processed or raw) and garlic (heat-processed) appeared to counter the decrease in oleic acid content by 10,18 and $20 \%$, respectively, compared to the LG diet group.

Table 3. Effect of feeding garlic and onion on serum lipid profile during cholesterol gallstone induction in micef

(Mean values and standard deviations for six samples per group, three mice per sample)

\begin{tabular}{|c|c|c|c|c|c|c|c|c|}
\hline \multirow[b]{2}{*}{ Diet group } & \multicolumn{2}{|c|}{$\begin{array}{l}\text { Total choles- } \\
\text { terol (mg/l) }\end{array}$} & \multicolumn{2}{|c|}{$\begin{array}{l}\text { Phospholipid } \\
\text { (mg/l) }\end{array}$} & \multicolumn{2}{|c|}{ TAG (mg/l) } & \multicolumn{2}{|c|}{$\mathrm{C} / \mathrm{PL}$} \\
\hline & Mean & SD & Mean & SD & Mean & SD & Mean & SD \\
\hline Control & 1855 & 231 & 3155 & 142 & 1495 & 919 & 0.58 & 0.07 \\
\hline LG & $4539^{*}$ & 320 & $1605^{*}$ & 151 & $1854^{*}$ & 113 & $2 \cdot 84^{\star}$ & 0.29 \\
\hline $\mathrm{LG}+$ garlic $(\mathrm{R})$ & $3048 \dagger$ & 281 & $2840 \dagger$ & 136 & 1720 & 93.9 & $1.07 \dagger$ & 0.11 \\
\hline $\mathrm{LG}+$ garlic $(\mathrm{H})$ & $3234 \dagger$ & 178 & $2799 \dagger$ & 148 & 1772 & 69.5 & $1.80 \dagger$ & $0 \cdot 17$ \\
\hline$L G+$ onion $(R)$ & $3143+$ & 178 & $2118 \dagger$ & 134 & 1737 & $67 \cdot 4$ & $1.48 \dagger$ & 0.12 \\
\hline$L G+$ onion $(\mathrm{H})$ & $2988 \dagger$ & 194 & $2870 \dagger$ & 186 & $1659 \dagger$ & 106 & $1.04 \dagger$ & 0.08 \\
\hline
\end{tabular}

$\mathrm{C} / \mathrm{PL}$, cholesterol:phospholipid ratio; $\mathrm{H}$, heat-processed; LG, lithogenic; R, raw.

Mean values were significantly different from those of the control group: ${ }^{*} P<0.01$.

Mean values were significantly different from those of the LG group: $† P<0.01$.

‡For details of diets and procedures, see Experimental methods. 
Table 4. Effect of feeding garlic and onion on liver lipid profile during cholesterol gallstone induction in miceł

(Mean values and standard deviations for six samples per group, three mice per sample)

\begin{tabular}{|c|c|c|c|c|c|c|c|c|}
\hline \multirow[b]{2}{*}{ Groups } & \multicolumn{2}{|c|}{$\begin{array}{l}\text { Total choles- } \\
\text { terol (mg/g) }\end{array}$} & \multicolumn{2}{|c|}{$\begin{array}{l}\text { Phospholipid } \\
\text { (mg/g) }\end{array}$} & \multicolumn{2}{|c|}{ TAG (mg/g) } & \multicolumn{2}{|c|}{$\mathrm{C} / \mathrm{PL}$} \\
\hline & Mean & SD & Mean & SD & Mean & SD & Mean & SD \\
\hline Control & 8.63 & 1.51 & $42 \cdot 7$ & $2 \cdot 29$ & $27 \cdot 0$ & 4.01 & 0.20 & 0.04 \\
\hline LG & $21 \cdot 3^{\star}$ & 2.07 & $30 \cdot 6^{\star}$ & $2 \cdot 66$ & $53 \cdot 7^{\star}$ & $2 \cdot 72$ & $0.69^{\star}$ & 0.15 \\
\hline $\mathrm{LG}+$ garlic $(\mathrm{R})$ & 16.0† & $2 \cdot 07$ & $39 \cdot 2 \dagger$ & $2 \cdot 10$ & $43.5 \dagger$ & $2 \cdot 84$ & $0.41 \dagger$ & $0 \cdot 10$ \\
\hline LG + garlic $(H)$ & $18 \cdot 1 \dagger$ & $1 \cdot 15$ & $34.7 \dagger$ & $2 \cdot 65$ & $47.5 \dagger$ & $2 \cdot 15$ & $0.52 \dagger$ & 0.14 \\
\hline$L G+$ onion $(R)$ & $18 \cdot 0 \dagger$ & $2 \cdot 04$ & $33.1 \dagger$ & $2 \cdot 04$ & $46 \cdot 3 \dagger$ & $1 \cdot 72$ & $0.54 \dagger$ & 0.09 \\
\hline$L G+$ onion $(\mathrm{H})$ & $14.9 \dagger$ & 2.40 & $42 \cdot 7 \dagger$ & 2.69 & $34.8 \dagger$ & $3 \cdot 19$ & $0.34 \dagger$ & 0.07 \\
\hline
\end{tabular}

C/PL, cholesterol:phospholipid ratio; $H$, heat-processed; LG, lithogenic; $R$, raw.

Mean values were significantly different from those of the control group: ${ }^{\star} P<0.01$.

Mean values were significantly different from those of the LG group: $† P<0.01$.

$\ddagger$ For details of diets and procedures, see Experimental methods.

\section{Effect of feeding garlic and onion on enzymes of cholesterol} metabolism during cholesterol gallstone induction

Activities of hepatic HMG-CoA reductase, the key regulatory enzyme involved in the biosynthesis of cholesterol and cholesterol $7 \alpha$-hydroxylase, the regulatory enzyme in the bile acid biosynthesis in experimental mice during CGS induction, are presented in Table 9. Feeding of LG diet reduced the hepatic HMG-CoA reductase activity significantly. This decrease in enzyme activity was countered by dietary garlic and onion to an extent of 92, 44, 61 and $35 \%$, respectively, in onion (heat-processed and raw) and garlic (heat-processed and raw) diet groups compared to the LG group. Activities of cholesterol $7 \alpha$-hydroxylase and sterol 27-hydroxylase were inhibited significantly by LG diet compared to the control group. Feeding of Allium spices along with LG diet enhanced the activity of both enzymes. Cholesterol $7 \alpha$-hydroxylase activity was increased by 59, 25, 18 and $37 \%$ in onion (heat-processed and raw) and garlic (heat-processed and raw) groups, respectively, compared to the LG group. Similarly, sterol 27-hydroxylase activity was increased in onion (heat-processed and raw) and garlic (heat-processed and raw) diet groups by $87,54,42$ and $80 \%$, respectively, when compared to the LG group.

\section{Discussion}

The present investigation used the well-studied mouse model for the induction of CGS by feeding high-cholesterol $\operatorname{diet}^{(26)}$. Optimal duration of feeding this LG diet for maximal CGS induction was found to be 10 weeks. Few common spices or their active principles have been shown to reduce biliary cholesterol and at the same time increase the bile acid concentration and water content in the bile ${ }^{(27-30)}$. The net result of these effects would be dilution of bile. Dilute biles are understood to prolong cholesterol nucleation times ${ }^{(31)}$. Previously, curcumin (colouring principle of turmeric) and capsaicin (pungent principle of red pepper) have been shown to prevent CGS formation in mice during experimental induction of the same ${ }^{(10,11)}$.

Allium spices, garlic and onion, have been known to possess health beneficial hypolipidaemic, hypocholesterolaemic and hydrocholagoguic influences ${ }^{(9)}$. The objective of the present study was therefore to evaluate the influence of garlic and onion on LG diet-induced CGS formation. Cooking or roasting alters the nature of many food constituents such as starches and proteins by changing their physical, chemical and nutritional properties. It also changes the bioavailability of proteins, carbohydrates, lipids and vitamins. It has been reported that boiling of spice mixes with food ingredients

Table 5. Effect feeding of garlic and onion on biliary lipids during cholesterol gallstone induction in miceł (Mean values and standard deviations for six samples per group, three mice per sample)

\begin{tabular}{|c|c|c|c|c|c|c|c|c|c|c|}
\hline \multirow[b]{3}{*}{ Diet group } & \multicolumn{6}{|c|}{ Biliary lipids (mM) } & & & & \\
\hline & \multicolumn{2}{|c|}{ Cholesterol } & \multicolumn{2}{|c|}{ Phospholipid } & \multicolumn{2}{|c|}{ Bile acids } & \multicolumn{2}{|c|}{ Total lipid (g/l) } & \multirow[b]{2}{*}{$\mathrm{C} / \mathrm{PL}$} & \multirow[b]{2}{*}{ C/BA } \\
\hline & Mean & SD & Mean & SD & Mean & SD & Mean & SD & & \\
\hline Control & 5.56 & 0.65 & $12 \cdot 3$ & $1 \cdot 79$ & $216 \cdot 4$ & $25 \cdot 1$ & 109 & $12 \cdot 9$ & 0.45 & 0.03 \\
\hline LG & $33 \cdot 5^{\star}$ & $3 \cdot 30$ & $21 \cdot 2^{*}$ & $3 \cdot 24$ & $228 \cdot 0$ & $15 \cdot 2$ & 114 & 9.5 & $1.58^{\star}$ & $0.15^{\star}$ \\
\hline $\mathrm{LG}+$ garlic $(\mathrm{R})$ & $13 \cdot 7 \dagger$ & 2.98 & 18.5 & 3.84 & 218.5 & $19 \cdot 2$ & 111 & $12 \cdot 4$ & $0.74 \dagger$ & $0.06 \dagger$ \\
\hline$L G+$ garlic $(H)$ & $21 \cdot 7 \dagger$ & 4.01 & $22 \cdot 5$ & 3.60 & $219 \cdot 2$ & $15 \cdot 9$ & 112 & 11.8 & $0.96 \dagger$ & $0.10 \dagger$ \\
\hline$L G+$ onion $(R)$ & $17.0 \dagger$ & $3 \cdot 60$ & $20 \cdot 2$ & 3.95 & $215 \cdot 3$ & 20.5 & 110 & $11 \cdot 7$ & $0.84 \dagger$ & $0.08 \dagger$ \\
\hline$L G+$ onion $(\mathrm{H})$ & $11.5 \dagger$ & 1.95 & $15 \cdot 7 \dagger$ & $3 \cdot 13$ & $222 \cdot 6$ & $19 \cdot 8$ & 113 & 8.4 & $0.73 \dagger$ & $0.05 \dagger$ \\
\hline
\end{tabular}

C/BA, cholesterol:bile acid ratio; $\mathrm{C} / \mathrm{PL}$, cholesterol:phospholipid ratio; $\mathrm{H}$, heat-processed; LG, lithogenic; $\mathrm{R}$, raw.

Mean values were significantly different from those of the control group: ${ }^{\star} P<0.01$.

Mean values were significantly different from those of the LG group: $† P<0.01$.

‡For details of diets and procedures, see Experimental methods. 
Table 6. Effect of feeding garlic and onion on biliary bile salt profile (mole fraction) during cholesterol gallstone induction in miceł (Mean values and standard deviations for six samples per group, three mice per sample)

\begin{tabular}{|c|c|c|c|c|c|c|c|c|c|c|c|c|}
\hline \multirow[b]{2}{*}{ Diet group } & \multicolumn{2}{|c|}{ TMC } & \multicolumn{2}{|c|}{ TUDC } & \multicolumn{2}{|c|}{ TC } & \multicolumn{2}{|c|}{ TCDC } & \multicolumn{2}{|c|}{ TDC } & \multicolumn{2}{|c|}{$\mathrm{GC}$} \\
\hline & Mean & SD & Mean & SD & Mean & SD & Mean & SD & Mean & SD & Mean & SD \\
\hline Control & 0.24 & 0.07 & 0.021 & 0.005 & 0.59 & 0.06 & 0.048 & 0.009 & 0.056 & 0.005 & 0.020 & 0.005 \\
\hline LG & $0.05^{\star}$ & 0.01 & $0.008^{*}$ & 0.002 & $0.73^{\star}$ & 0.08 & $0.040^{*}$ & 0.012 & $0.135^{\star}$ & 0.029 & $0.020^{*}$ & 0.009 \\
\hline LG + garlic (R) & $0.19 \dagger$ & 0.02 & $0.015 \dagger$ & 0.005 & $0.63 \dagger$ & 0.05 & $0.045 \dagger$ & 0.01 & $0.091 \dagger$ & 0.022 & $0.020 \dagger$ & 0.003 \\
\hline$L G+\operatorname{garlic}\left(H^{\prime}\right)$ & $0.15 \dagger$ & 0.02 & $0.013 \dagger$ & 0.003 & $0.65 \dagger$ & 0.09 & $0.045 \dagger$ & 0.006 & $0.111 \dagger$ & 0.009 & $0.022 \dagger$ & 0.002 \\
\hline $\mathrm{LG}+$ onion $(\mathrm{R})$ & $0.19 \dagger$ & 0.03 & $0.013 \dagger$ & 0.003 & $0.62 \dagger$ & 0.11 & $0.041 \dagger$ & 0.012 & $0.104 \dagger$ & 0.013 & $0.021 \dagger$ & 0.009 \\
\hline$L G+$ onion $(H)$ & $0.21 \dagger$ & 0.03 & $0.015 \dagger$ & 0.005 & $0.59 \dagger$ & 0.12 & $0.038 \dagger$ & 0.013 & $0.115 \dagger$ & 0.014 & $0.018 \dagger$ & 0.007 \\
\hline
\end{tabular}

GC, glycocholic acid; H, heat-processed; LG, lithogenic; R, raw; TC, taurocholic acid; TCDC, taurochenodexoycholic acid; TDC, taurodeoxycholic acid; TMC, tauromuricholic acid; TUDC, tauroursodexoycholic acid.

Mean values were significantly different from those of the control group: ${ }^{*} P<0.01$.

Mean values were significantly different from those of the $L G$ group: $† P<0.01$.

$\ddagger$ For details of diets and procedures, see Experimental methods.

results in the loss of active principles of common spices during domestic cooking ${ }^{(12)}$. The present study also evaluated the effect of heat-treatment of the test spices on their antilithogenic potential. Earlier studies in our laboratory with rats have indicated that $2 \%$ dietary onion or $0.5 \%$ dietary garlic powder produced significant increase in bile volume and in the secretion of biliary bile acids presumably due to higher rate of conversion from cholesterol ${ }^{(29,30)}$. Such a situation would contribute to decreasing the CSI in the bile. These dietary doses of the two Allium spices were hence examined in the present study for an antilithogenic influence during high cholesterol feeding in experimental mice. These doses of garlic and onion roughly correspond to five times what is generally consumed by the population in India ${ }^{(32)}$.

Feeding of LG diet significantly increased liver weight due to high cholesterol deposition and higher TAG content. Feeding of garlic and onion partially countered this increase in liver weights obviously by correcting the alterations in the hepatic lipid profile. Feeding of LG diet to mice caused secretion of more cholesterol molecules into bile leading to the formation of lithogenic bile. LG diet feeding for 10 weeks consistently induced CGS formation in all animals. Inclusion of raw/heat-processed garlic or onion in the diet

Table 7. Effect of dietary garlic and onion on cholesterol saturation index (CSI) and hydrophobicity index (HI) of bile during cholesterol gallstone induction in miceł

(Mean values and standard deviations for six samples per group, three mice per sample)

\begin{tabular}{llllll}
\hline & \multicolumn{2}{c}{ CSI } & & \multicolumn{2}{c}{$H I$} \\
\cline { 2 - 3 } \cline { 5 - 6 } Diet group & Mean & SD & & Mean & SD \\
\hline Control & 0.51 & 0.07 & & -0.145 & 0.015 \\
LG & $1.90^{*}$ & 0.25 & & $+0.054^{*}$ & 0.012 \\
LG + garlic (R) & $0.92 \dagger$ & 0.13 & & $-0.079 \dagger$ & 0.013 \\
LG + garlic (H) & $1.25 \dagger$ & 0.13 & & $-0.032 \dagger$ & 0.002 \\
LG + onion (R) & $1.09 \dagger$ & 0.13 & & $-0.073 \dagger$ & 0.008 \\
LG + onion (H) & $0.86 \dagger$ & 0.16 & $-0.088 \dagger$ & 0.021 \\
\hline
\end{tabular}

$H$, heat-processed; LG, lithogenic; $R$, raw.

Mean values were significantly different from those of the control group: ${ }^{\star} P<0.01$

Mean values were significantly different from those of the LG group: $\dagger P<0.01$.

$\ddagger$ For details of diets and procedures, see Experimental methods. significantly reduced the incidence of CGS. The pathophysiological conditions that might be responsible for the cholesterol crystallization and gallstone formation is increased cholesterol content and decreased bile acids and phospholipids in bile. It has been reported that mice susceptible to gallstones secrete more cholesterol into bile at canalicular membrane level leading to the formation of lithogenic bile ${ }^{(33)}$. This leads to higher CSI resulting in the nucleation of cholesterol leading to the formation of CGS. The LG diet feeding in the present study increased the hepatic cholesterol, a significant part of which is secreted into bile.

The supersaturation of bile with cholesterol (CSI $>1)$ leads to the formation of $\mathrm{CGS}^{(34)}$. The CSI in the spice-fed groups is $<1$ indicating that the cholesterol content of bile is low and bile acid and phospholipid contents are more compared to the LG diet-fed group. The inclusion of Allium spices in the diet reduced the incidence of CGS formation and is evident where the CSI $<1$. The CSI in spice-fed animals was much lower than that of the LG group which means that the bile of these animals has the capacity to solubilize more cholesterol due to higher bile acid and phospholipid concentration present in it relative to cholesterol concentration. The present results also indicated that CGS is present in mice even when the CSI $<1$ and the effect of these spices is only partial. It has been shown that CSI being $>1$ is a prerequisite for the precipitation of cholesterol and subsequent stone formation but it is not always true as in some cases CGS were observed when CSI $<1$. This is because there may be several other factors that are responsible for the formation of CGS. Cholesterol secretion into bile is tightly coupled to phospholipid and bile acid secretion $^{(35)}$.

Biliary cholesterol/phospholipid secretion rates are considered to be closely linked to bile acid secretion rates and enhanced by higher bile acids in bile. The C/PL ratio of bile indicates the C/PL ratio of the vesicles whose secretion is induced by bile acids at the canaliculus ${ }^{(33)}$. In the present study, LG diet feeding significantly increased C/PL ratio which paralleled the elevated CSI in bile. In contrast, animals maintained on spice diets showed a significant decrease in C/PL and CSI compared to the LG diet group. This shows that LG diet-fed mice secrete more cholesterol into bile at the canalicular membrane level which is a major factor leading to the formation of lithogenic bile. The present results are also in agreement with Hoffmann \& Grundy ${ }^{(36)}$ who observed that there was a high C/PL 
Table 8. Effect of dietary garlic and onion on fatty acid composition of biliary phospholipids (\% total fatty acids) during cholesterol gallstone induction in miceł

(Mean values and standard deviations for six samples per group, three mice per sample)

\begin{tabular}{|c|c|c|c|c|c|c|c|c|c|c|c|c|}
\hline \multirow[b]{2}{*}{ Diet group } & \multicolumn{2}{|c|}{$16: 0$} & \multicolumn{2}{|c|}{$16: 1$} & \multicolumn{2}{|c|}{$18: 0$} & \multicolumn{2}{|c|}{$18: 1$} & \multicolumn{2}{|c|}{$18: 2$} & \multicolumn{2}{|c|}{$20: 4$} \\
\hline & Mean & SD & Mean & SD & Mean & SD & Mean & SD & Mean & SD & Mean & SD \\
\hline Control & $16 \cdot 9$ & 1.12 & 5.33 & 0.21 & 3.72 & 0.18 & $62 \cdot 3$ & $2 \cdot 31$ & $7 \cdot 51$ & 0.54 & 4.09 & 0.21 \\
\hline LG & $25 \cdot 3^{\star}$ & 1.89 & $7 \cdot 50^{\star}$ & 1.05 & 3.38 & 0.14 & $47 \cdot 2^{*}$ & $3 \cdot 21$ & $10 \cdot 3^{*}$ & 1.32 & 4.04 & 0.21 \\
\hline$L G+$ garlic $(R)$ & $25 \cdot 9$ & $2 \cdot 31$ & $7 \cdot 68$ & 1.56 & 3.46 & 0.23 & $48 \cdot 3$ & $3 \cdot 13$ & $10 \cdot 5$ & 0.89 & $4 \cdot 14$ & 0.32 \\
\hline$L G+\operatorname{garlic}(H)$ & $21 \cdot 3$ & $3 \cdot 11$ & $3.00 \dagger$ & 0.53 & $1.84 \dagger$ & 0.14 & $56.5 \dagger$ & $4 \cdot 16$ & $9 \cdot 24$ & 0.98 & $8.08 \dagger$ & 0.65 \\
\hline $\mathrm{LG}+$ onion $(\mathrm{R})$ & $20 \cdot 1 \dagger$ & $1 \cdot 19$ & $2 \cdot 84 \dagger$ & $0 \cdot 14$ & $5.45 \dagger$ & 0.34 & $56.0 \dagger$ & $4 \cdot 16$ & 9.69 & 1.21 & $5 \cdot 90$ & 0.65 \\
\hline $\mathrm{LG}+$ onion $(\mathrm{H})$ & $22 \cdot 1$ & 2.01 & $3.21 \dagger$ & 0.31 & 4.86 & $3 \cdot 21$ & $52 \cdot 1 \dagger$ & 3.16 & $8 \cdot 78$ & 1.65 & $8.90 \dagger$ & 0.31 \\
\hline
\end{tabular}

$H$, heat-processed; LG, lithogenic; $R$, raw.

Mean values were significantly different from those of the control group: ${ }^{*} P<0.01$

Mean values were significantly different from those of the $L G$ group: $\dagger P<0 \cdot 01$.

$\ddagger$ For details of diets and procedures, see Experimental methods.

ratio in the bile of CGS patients. This suggests that in CGS patients as well as in mice nascent phospholipid vesicles at the canalicular level may be enriched with cholesterol and the test spices prevented this enrichment.

The C/PL ratio and cholesterol:bile acids ratio were significantly increased upon feeding LG diet due to increased cholesterol in bile. Dietary garlic or onion reduced these ratios significantly compared to the LG diet group. Bile acids provide primary stimulus for bile flow and facilitate the secretion of lipids into bile ${ }^{(37,38)}$. Biliary secretion of phospholipids is dependent on the bile acids. During bile formation, bile acids stimulate secretion of phospholipids from hepatocytes $^{(39)}$. Increased secretion of cholesterol is related to phospholipids with more hydrophobic molecular species of phospholipids in the bile ${ }^{(35)}$. Compared to the control group, the total bile acid content of bile in the LG group was increased slightly whereas the cholesterol content increased five-fold. On the other hand, feeding of spices significantly decreased the biliary cholesterol and increased the bile acids, though making the bile slightly more hydrophilic. Phospholipid content was also increased relatively compared to cholesterol in the LG group and in spice-fed animals. The C/PL ratio was significantly increased in th $L G$ group, which was countered by feeding spices. Increases in the bile acids, bile flow and phospholipids help in solubilization of cholesterol in mixed micelles and vesicles, the major carriers of cholesterol in bile. This stabilized the bile and increased the solubilization of cholesterol and prolonged the cholesterol nucleation time. It has been shown that due to concentration of bile in the gallbladder by absorption of water, elevation of biliary vesicular C/PL ratio occurs leading to vesicular aggregation, which precedes nucleation ${ }^{(31)}$. As the bile gets concentrated in the gallbladder, the vesicles become rich in cholesterol, resulting in an increased C/PL ratio in vesicles. With this increased ratio, the vesicles become unstable and tend to nucleate cholesterol ${ }^{(40)}$.

The hepatobiliary pathway is the major route for the removal of cholesterol from the body either after converting into bile acids or in the form of free cholesterol. Dietary cholesterol inhibits hepatic cholesterol synthesis, which in turn impairs bile acid synthesis. These three factors lead to supersaturation of bile with cholesterol (CSI $>1)$. Bile with higher CSI $(>1)$ is prone to cholesterol crystal nucleation. Hence biliary lipid secretion plays an important role in cholesterol homeostasis.

Bile acids are natural detergents that solubilize lipids in the intestinal tract and in bile. The relative balance of hydrophilic and hydrophobic bile acids in the bile depends upon their state

Table 9. Effect of feeding garlic and onion on hepatic cholesterol-metabolizing enzymes in miceł

(Mean values and standard deviations for five samples per group, three mice per sample)

\begin{tabular}{|c|c|c|c|c|c|c|}
\hline \multirow[b]{2}{*}{ Diet group } & \multicolumn{2}{|c|}{$\begin{array}{l}\text { HMG-CoA reductase } \\
\text { (nmol CoA formed/- } \\
\text { min per mg protein) }\end{array}$} & \multicolumn{2}{|c|}{$\begin{array}{c}\text { Cholesterol } \\
7 \alpha \text {-hydroxylase (pmol } \\
7 \alpha \text {-hydroxycholesterol } \\
\text { formed } / \text { min } \\
\text { per mg protein) }\end{array}$} & \multicolumn{2}{|c|}{$\begin{array}{l}\text { Sterol 27-hydroxylase } \\
\text { (pmol 27-hydroxy } \\
\text { cholesterol } \\
\text { formed/min } \\
\text { per mg protein) }\end{array}$} \\
\hline & Mean & SD & Mean & SD & Mean & SD \\
\hline Control & $67 \cdot 4$ & 4.38 & $14 \cdot 6$ & 1.96 & 48.9 & $5 \cdot 11$ \\
\hline LG & $28 \cdot 6^{\star}$ & $4 \cdot 31$ & $3 \cdot 86^{\star}$ & 0.73 & $16 \cdot 4^{*}$ & $2 \cdot 13$ \\
\hline $\mathrm{LG}+\operatorname{garlic}(\mathrm{R})$ & $38.7 \dagger$ & $2 \cdot 81$ & $5 \cdot 30 \dagger$ & 1.20 & $29.4 \dagger$ & 3.18 \\
\hline $\mathrm{LG}+\operatorname{garlic}(\mathrm{H})$ & $46 \cdot 2 \dagger$ & 5.63 & $4.56 \dagger$ & 0.81 & $23.3 \dagger$ & 4.73 \\
\hline $\mathrm{LG}+$ onion $(\mathrm{R})$ & $41 \cdot 3 \dagger$ & 2.98 & $4.82 \dagger$ & $1 \cdot 12$ & $25 \cdot 2 \dagger$ & 3.88 \\
\hline$L G+$ onion $(H)$ & $54.9 \dagger$ & $6 \cdot 78$ & $6 \cdot 14 \dagger$ & 0.64 & $30.6 \dagger$ & 2.98 \\
\hline
\end{tabular}

$\mathrm{H}$, heat-processed; HMG, hydroxymethyl glutaryl; LG, lithogenic; R, raw.

Mean values were significantly different from those of the control group: ${ }^{\star} P<0.01$.

Mean values were significantly different from those of the LG group: $\dagger P<0.01$.

$\ddagger$ For details of diets and procedures, see Experimental methods. 
of ionization, number, position and orientation of hydroxyl groups, and by the presence and nature of ring or side-chain esters. The bile acids taurocholic acid, tauromuricholic acid and tauroursodeoxycholate acid are termed hydrophilic bile salts. Taurochenodeoxycholic acid and taurodeoxycholic acid are termed hydrophobic bile acids. Feeding of garlic or onion significantly increased the hydrophilic bile acids such as taurocholic acid, tauromuricholic acid and tauroursodeoxycholate acid. Earlier it has been reported that increased content of biliary deoxycholic acid in CGS patients or increased deoxycholic acid content in bile promotes rapid cholesterol crystallization and formation of CGS. In fact, it was demonstrated that deoxycholic acid is highly hydrophobic and was associated with human cholelithiasis ${ }^{(41)}$. The hydrophobicity of bile acids plays an important role in gallstone formation. It is suggested that glycine-conjugated bile acids are more hydrophobic which are lithogenic in nature, while taurine conjugates have opposite effects. It is understood that bile acids are conjugated more with taurine in rodents when the supply of taurine is sufficient, while glycine-conjugated bile salts are produced when taurine is deficient ${ }^{(42)}$. The fatty acid composition of phospholipid exhibited significant influence on CSI in monkeys and man, with a particular negative relationship with linoleic acid ${ }^{(43)}$. In the present study, linoleic acid and oleic acid content were increased by feeding spices as compared to the LG diet.

Biosynthesis of cholesterol is regulated by HMG-CoA reductase activity, whereas its biodegradation to bile acids in hepatocytes is carried by cholesterol $7 \alpha$-hydroxylase and sterol 27-hydroxylase ${ }^{(44)}$. Hepatic HMG-CoA reductase activity was diminished by LG diet due to negative feedback inhibition. Feeding Allium spices could restore this altered activity significantly. The possible explanation for this can be that cholesterol synthesized by de novo process might be preferred over the exogenous cholesterol for conversion into bile acids and for further excretion in bile. Similarly, cholesterol $7 \alpha$-hydroxylase and sterol 27 -hydroxylase activity were inhibited by feeding LG diet, while garlic and onion countered this decreased enzyme activity significantly. Earlier, we have reported that cholesterol $7 \alpha$-hydroxylase activity in rats was increased by feeding spices ${ }^{(45)}$. It has been evidenced earlier that in exercising the hypocholesterolaemic effect, garlic does not influence the intestinal absorption of cholesterol, and it is also documented that dietary garlic does not cause higher faecal excretion of cholesterol or bile acids ${ }^{(46)}$.

The increase in serum and liver cholesterol levels was reflected in the increased CSI in bile and CGS occurrence in LG diet-fed animals. Feeding garlic or onion significantly brought down the cholesterol content in serum and liver and it was evident in the lowered CSI values and decreased CGS occurrence. The increased $\mathrm{C} / \mathrm{PL}$ ratios in serum and liver were reflected in the higher CSI and CGS occurrence. The spice diets significantly brought down this ratio, which was reflected in the lower CSI and CGS occurrence. The lowered levels of cholesterol may be the combined result of decreased synthesis (as reflected in decreased HMG-CoA reductase activity) and increased conversion to bile acids (as reflected in increased cholesterol $7 \alpha$-hydroxylase and sterol 27 -hydroxylase activity). The sulphur compounds, alliin and allicin of garlic, and propenyl sulphides present in onion, are presumably responsible for the hypocholesterolaemic influences of the parent spices ${ }^{(9)}$. The antilithogenicity of garlic and onion is attributable primarily to this hypocholesterolaemic influence produced by these bioactive sulphur compounds.

The present study clearly demonstrated that feeding of garlic and onion inhibited the formation of CGS in mice during experimental induction by decreasing the cholesterol content in serum, liver and bile. This is also reflected in the lowering of CSI in the bile of these mice. The present study also showed that heat processing of these Allium spices does not significantly compromise the antilithogenic influence, and in fact heat-processed onion was even more effective than its raw form in alleviating the incidence of CGS in mice. The dietary dosage of garlic and onion evaluated here which is roughly five times what is normally consumed can probably be tolerated to derive this health benefit. Although a combination of phenotypic expression of genetic traits predisposes to stone formation, the higher prevalence of CGS disease in the USA, Chile and Europe (Sweden, Germany and Austria) relative to Asian and African countries and the very high prevalence in ethnic groups such as American Indians and Chilean Mapuche Indians could probably be mitigated by the liberal consumption of these Allium spices.

\section{Acknowledgements}

S. V. thanks the Indian Council of Medical Research, New Delhi, India for the award of a Senior Research Fellowship. This work was supported by grant-in-aid from the Department of Science and Technology, Government of India, New Delhi. There are no conflicts of interest whatsoever among the authors. S. V. is responsible for the bench work, K. Sambaiah closely supervised the whole animal experiment and analytical work. K. Srinivasan is the project leader, who handled research planning, data interpretation and writing of the manuscript.

\section{References}

1. Johnston DE \& Kaplan MM (1993) Pathogenesis and treatment of gallstones. $N$ Engl J Med 328, 412-421.

2. Juvonen T (1994) Pathogenesis of gallstones. Scand J Gastroenterol 29, 577-582.

3. Apstein MD \& Carey MC (1996) Pathogenesis of cholesterol gallstones - a parsimonious hypothesis. Eur J Clin Invest 26, 343-352.

4. Marzolo MP, Rigotti A \& Nervi F (1990) Secretion of biliary lipids from the hepatocyte. Hepatology 12, Suppl., 134-142.

5. Ozben T (1989) Biliary lipids, composition and gallstone formation in rabbits fed on soya protein, cholesterol, casein and modified casein. Biochem J 263, 293-296.

6. Scobey MW, Johnson FL, Parks JS, et al. (1991) Dietary fish oil effects on biliary lipid secretion and cholesterol gallstone formation in the African green monkeys. Hepatology 14, 679-684.

7. Judd PA (1985) Dietary fibre and gallstones. In Dietary Fibre Perspective: Reviews and Bibliography, pp. 40-46 [AR Leeds, editor]. London: John Libbey.

8. Ebihara K \& Kiriyama S (1985) Prevention of CGS by a water soluble fibre - Konjac Mannan in mice. Nutr Rep Int 32, 223-229.

9. Srinivasan K, Sambaiah K \& Chandrasekhara N (2004) Spices as beneficial hypochole-sterolemic food adjuncts: a review. Food Rev Int 20, 187-220. 
10. Hussain MS \& Chandrasekhara N (1993) Influence of curcumin and capsaicin on cholesterol gallstone induction in hamsters and mice. Nutr Res 13, 349-357.

11. Hussain MS \& Chandrasekhara N (1994) Effect of curcumin and capsaicin on the regression of pre-established CGS in mice. Nutr Res 14, 1561-1574.

12. Suresh D, Manjunatha H \& Srinivasan K (2007) Effect of heat processing of spices on the concentrations of their bioactive principles: turmeric (Curcuma longa), red pepper (Capsicum annuum) and black pepper (Piper nigrum). J Food Comp Anal 20, 346-351.

13. Bligh EG \& Dyer WJ (1959) A rapid method of total lipid extraction and purification. Can J Biochem Physiol 37, 911-917.

14. Turley SD \& Dietschy JM (1970) Reevaluation of $3 \alpha$-hydroxysteroid dehydrogenase assay for total bile acids. J Lipid Res 19, 924-928.

15. Folch J, Lees M \& Sloane-Stanley GH (1957) A simple method for the isolation and purification of total lipids from animal tissue. J Biol Chem 226, 497-509.

16. Searcy RL \& Bergquist LM (1960) A new colour reaction for the quantification of serum cholesterol. Clin Chim Acta 5, 192-199.

17. Charles J \& Stewart M (1980) Colorimetric estimation of phospholipids with ammonium ferrothiocyanate. Anal Biochem 104, 10-14.

18. Fletcher MJ (1968) A colorimetric method for estimating serum triglycerides. Clin Chim Acta 22, 303-307.

19. Rosi SS, Converse JL \& Hofmann AF (1987) High pressure liquid chromatographic analysis of conjugated bile acids in human bile: simultaneous resolution of sulfated and unsulfated lithocholyl amidates and the common conjugated bile acids. J Lipid Res 28, 589-595.

20. Heuman DM (1989) Quantitative estimation of the hydrophilichydrophobic balance of mixed bile salt solutions. J Lipid Res 30, 719-730.

21. Carey MC (1978) Critical tables for calculating the cholesterol saturation of native bile. J Lipid Res 19, 945-955.

22. Morrison MR \& Smith M (1964) Preparation of fatty acid methyl esters and dimethyl acetyls from lipids with boron trifluoride methanol. J Lipid Res 5, 600-608.

23. Hulcher FH \& Oleson WH (1973) Simplified spectrophotometric assay for microsomal 3-hydroxy-3-methylglutaryl coenzyme A reductase by measurement of coenzyme-A. J Lipid Res 14, 625-631.

24. Petrack B \& Latario BJ (1993) Synthesis of 27-hydroxycholesterol in rat liver mitochondria: HPLC assay and marked activation by exogenous cholesterol. J Lipid Res 34, 643-649.

25. Lowry $\mathrm{OH}$, Rosebrough NJ, Farr AL, et al. (1951) Protein measurement with the Folin phenol reagent. J Biol Chem 193, $265-275$.

26. Tepperman J, Cladwell FT \& Tepperman HM (1964) Induction of gallstones in mice by feeding a cholesterol and cholic acid containing diet. Am J Physiol 206, 628-638.

27. Bhat GB, Srinivasan MR \& Chandrasekhara N (1984) Influence of curcumin and capsaicin on bile secretion and composition in the rat. J Food Sci Technol 21, 225-227.

28. Bhat GB, Sambaiah K \& Chandrasekhara N (1985) The effect of feeding fenugreek and ginger on bile composition in the albino rat. Nutr Rep Int 32, 1145-1151.
29. Sambaiah K \& Srinivasan K (1991) Secretion and composition of bile in rats fed diets containing spices. J Food Sci Technol $\mathbf{2 8}$, $35-38$.

30. Platel K \& Srinivasan K (2000) Stimulatory influence of select spices on bile secretion in rats. Nutr Res 20, 1493-1503.

31. van Erpecum KJ \& van Berge-Henegouwen GP (1989) Pathogenic factors in cholesterol gallstone disease. Scand J Gastroenterol 24, Suppl., 83-90.

32. Thimmayamma BVS, Rao P \& Radhaiah G (1983) Use of spices and condiments in the dietaries of urban and rural families. Indian J Nutr Dietet 20, 153-162.

33. Wang DQH, Lammert F, Paigen B, et al. (1999) Phenotypic characterization of lith genes that determine susceptibility to cholesterol cholelithiasis in inbred mice: pathophysiology of biliary lipid secretion. J Lipid Res 40, 2066-2079.

34. Carey MC \& Small DM (1978) The physical chemistry of cholesterol solubility in bile: relationship to gallstone formation and dissolution in man. $J$ Clin Invest 61, 998-1026.

35. Robins SJ, Fasulo JM, Robins VF, et al. (1991) Utilization of different fatty acids for hepatic and biliary PC formation and the effect of changes in PC molecular species on biliary lipid structures. J Lipid Res 32, 985-992.

36. Hoffmann AF \& Grundy SM (1982) Pretreatment biliary lipid composition in white patients with radiolucent gallstones in the National Cooperative Gallstone Study. Gastroenterology 83, 738-752.

37. Knox R, Stein I, Levinson D, et al. (1991) Effect of fat prefeeding on bile flow and composition in the rat. Biochim Biophys Acta 1083, 65-70.

38. Tierney S, Ahrendt SA, Fox K, et al. (1993) Fish oil reduces biliary cholesterol and prolongs nucleation of human gallbladder bile. Gastroenterology 104, 380-384.

39. Cohen DE, Angelico M \& Carey MC (1990) Structural alteration in lecithin-cholesterol vesicles following interactions with monomeric and micellar bile salts: physical-chemical basis for sub selection of biliary lipids during bile formation. J Lipid Res 31, 55-70.

40. Roslyn JJ, Doty J, Pitt HA, et al. (1986) Enhanced gallbladder absorption during gallstone formation: the roles of cholesterol saturated bile and gallbladder stasis. Am J Med Sci 292, 75-80.

41. Afdhal NH \& Smith BF (1990) Cholesterol crystal nucleation: a decade long search for the missing link in gallstone pathogenesis. Hepatology 11, 699-702.

42. Armstrong MJ \& Carey MC (1982) The hydrophobic-hydrophilic balance of bile salts: inverse correlation between reversephase high performance liquid chromatographic metabolites and micellar cholesterol solubilizing capacities. J Lipid Res 23, 70-80.

43. Berr F, Holl J, Jungst D, et al. (1992) Dietary $n$-3 polyunsaturated fatty acids decrease biliary cholesterol saturation in gallstone disease. Hepatology 16, 960-967.

44. Dietschy JM, Turley SD \& Spady DK (1993) Role of liver in the maintenance of cholesterol and low density lipoprotein homeostasis in different animal species, including humans. J Lipid Res 34, 1637-1659.

45. Srinivasan K \& Sambaiah K (1991) The effect of spices on cholesterol $7 \alpha$-hydroxylase activity and on serum hepatic cholesterol levels in the rats. Int J Vit Nutr Res 61, 364-369.

46. Kamanna VS \& Chandrasekhara N (1984) Hypocholesterolemic activity of different fractions of garlic. Indian J Med Res $\mathbf{9}$, $580-583$. 